Article

\title{
Frequency Difference Thermally and Electrically Tunable Dual-Frequency Nd:YAG/LiTaO 3 Microchip Laser
}

\author{
Kun Gui $\oplus^{\circ}$, Zilong Zhang *, Yuxiao Xing, Haiyang Zhang and Changming Zhao * \\ Beijing Institute of Technology, School of Optics and Photonics, 5 South Zhongguancun Street, \\ Beijing 100081, China; 2120160618@bit.edu.cn (K.G.); 3220180381@bit.edu.cn (Y.X.); ocean@bit.edu.cn (H.Z.) \\ * Correspondence: zlzhang@bit.edu.cn (Z.Z.); zhaochangming@bit.edu.cn (C.Z.); \\ Tel.: +86-010-6891-8861 (Z.Z. \& C.Z.)
}

Received: 13 March 2019; Accepted: 9 May 2019; Published: 14 May 2019

\begin{abstract}
This study presents a dual-frequency microchip laser with a thermo-optically and electro-optically tuned frequency difference. The dual-frequency microchip cavity is formed by bonding a Lithium tantalite $\left(\mathrm{LiTaO}_{3}, \mathrm{LTO}\right)$ crystal chip and a neodymium-doped yttrium aluminum garnet (Nd:YAG) crystal chip. A single longitudinal mode is generated by the Nd:YAG crystal and split into two frequencies with perpendicular polarizations due to birefringent effect in the LTO chip. Furthermore, continuous beat frequency tuning at different scales is realized by adjusting the temperature and voltage applied to the LTO crystal. A maximum beat frequency of up to 27 $\mathrm{GHz}$ is obtained, and the frequency difference lock-in phenomenon is observed below the frequency difference of $405 \mathrm{MHz}$.
\end{abstract}

Keywords: microwave photonics; tunable dual frequency; microchip laser; electro-optic effect; thermo-optic effect

\section{Introduction}

Frequency difference tunable dual-frequency lasers have drawn a lot of attention in the last few years for their applications in the fields of absolute distance interferometry, Light Detection and Ranging (LIDAR) detection and terahertz wave generation [1-3]. In recent years, significant achievements have been made in the research of dual-frequency lasers, such as the intensity balance ratio, beat note stability and beat effect-based Q-switch regime [4-6]. These achievements have led to dual-frequency laser technology with a complete system and rich connotation. LIDAR with a tunable dual-frequency laser as the light source has a better performance, for that the range and accuracy of speed measurement can be greatly improved. If the beat frequency can be tuned according to a target's speed, one can get the proper Doppler frequency shift which is more convenient for signal processing. If the beat frequency reaches several $\mathrm{GHz}$ or higher, the moving speed of $\mathrm{m} / \mathrm{s}$ or even $\mathrm{mm} / \mathrm{s}$ can be measured easier $[7,8]$. As well as in terms of distance measurement, with an ability to change the wavelength of the beat signal and realize high-resolution detection of targets at different distances $[9,10]$. Therefore, the tuning capability of frequency difference is an important property of the dual-frequency laser. 
The principle of birefringence is a common method implemented to split a single-frequency into a dual-frequency. For example, using two quarter-wave plates, one can continuously tune the frequency difference from a few gigahertz to $150 \mathrm{GHz}[11,12]$. However, the inaccurate rotating of the quarter wave plate hampers its application under circumstances where high precision is required. Mechanical stress-induced birefringence can generate a several $\mathrm{GHz}$ beat frequency signal with good linear relation. However, the beat frequency is not stable enough and is susceptible to external forces. It is necessary to pay attention to the pressure to avoid crushing the crystal, which also limits the range of frequency difference adjustment with this method $[5,13]$. Changing the temperature of the birefringent crystal can change its refractive index, which can tune the frequency difference over a wide range from 0 to 60 $\mathrm{GHz}$ [14]. Using the electro-optical effect of the crystal, an external electric field can be applied to tune the frequency difference with a short response time $[15,16]$. However, to achieve the few gigahertz frequency difference tuning, usually an applied voltage of several thousand volts is required.

This paper presents a dual-frequency microchip laser with a frequency difference that can be tuned by varying the cooling temperature and voltage applied to the laser. With a wide gain bandwidth, the Nd:YAG microchip is used as the gain medium to realize broad-range tuning of the beat frequency. LTO, as the birefringent crystal, has good thermal stability and a large electro-optical coefficient, making it an ideal candidate for dual-frequency generation. The single-frequency generated by the Nd:YAG is split into two perpendicularly polarized components by the LTO crystal. Furthermore, their frequency difference is first large-scale adjusted by controlling the cooling temperature, then fine-scale adjusted by the variable voltage applied to the LTO crystal. Combined with temperatureand voltage-tuning methods, it can ensure broad-range and high-accuracy tuning of the frequency difference simultaneously.

\section{Experimental Setup}

The schematic diagram of the dual-frequency microchip laser and its relevant measurement method are shown in Figure 1. The composite laser cavity consists of a $1 \%$ at doped $\mathrm{Nd}^{3+}:$ YAG gain medium chip with a $0.4 \mathrm{~mm}$ thickness and an LTO electro-optic crystal with $1 \mathrm{~mm}$ thickness. The two chips both have transverse dimensions of $5 \times 5 \mathrm{~mm}^{2}$, and are combined by the optical cement method. The surface of the Nd:YAG crystal is coated with a film that has high reflectivity ( $\mathrm{R}>99.7 \%)$ at $1064 \mathrm{~nm}$ and high transmissivity ( $\mathrm{T}>97 \%$ ) at $808 \mathrm{~nm}$, which serves as the end mirror of the cavity. The surface of LTO is coated with a film of high reflectivity $(\mathrm{R}=99 \%)$ at $1064 \mathrm{~nm}$, which serves as the output coupling mirror. An $808 \mathrm{~nm}$ diode laser coupled with an optical fiber (fiber core diameter is $105 \mu \mathrm{m}, \mathrm{NA}=0.22$ ) is used as the pump source to match one of the $\mathrm{Nd}^{3+}$ ion absorption peaks. The pump beam is focused into the Nd:YAG crystal with a spot size of about $100 \mu \mathrm{m}$ in diameter. The dual-frequency beam with orthogonal polarizations can be generated by virtue of the birefringence of LTO. A thermoelectric cooler (TEC) is used to control the temperature of the microchip laser. The lateral surface of LTO is coated with gold film, on which two electrodes are glued with conductive adhesive to apply a transverse electric field by a constant voltage source. The dual-frequency beam passes through the polarizer to generate a beat note. A high reflection (HR) mirror for the laser beam can also split pump light. The longitudinal modes could be measured by a Fabry-Perot (F-P) scanning interferometer. And the optical spectrum or beat frequency are measured by an optical spectrum analyzer or a radio frequency (RF) analyzer. 


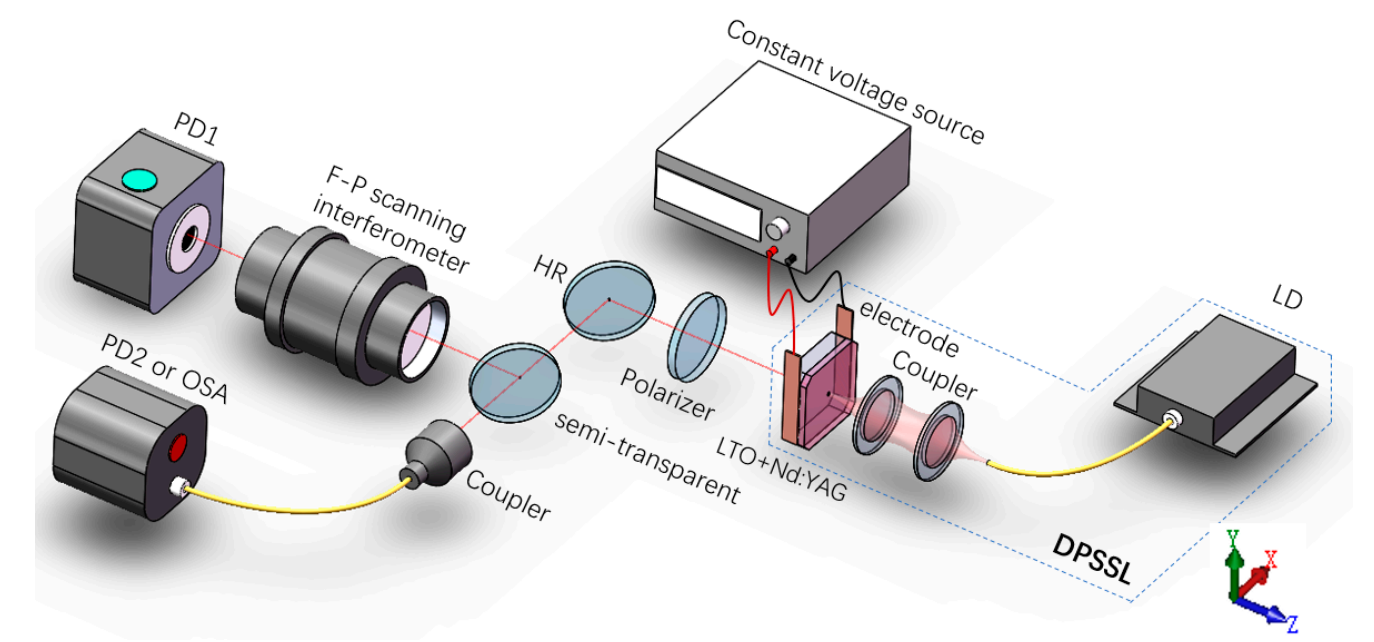

Figure 1. Schematic diagram of the experimental setup. LD, laser diode; HR, high-reflective mirror (@1064nm); PD, photoelectric detector; OSA, optical spectrum analyzer; DPSSL, diode-pumped solid-state laser.

\section{Theoretical Analysis}

In the microchip cavity, the stable oscillating laser satisfies the standing wave condition and has an optical frequency of:

$$
v_{q}=q \frac{c}{2 L}
$$

where $c$ is the velocity of light, $L$ is the optical path of the cavity, and $q$ is a natural number. The longitudinal mode interval is $\frac{c}{2 L}$. The optical path of the cavity consists of $L_{Y A G}=n_{Y A G} \times l_{Y A G}$ and $L_{L T O}=n_{L T O} \times l_{L T O}$. The refractive index of Nd:YAG is 1.82, and for LTO, the mean value is 2.14. Then we can get a longitudinal mode interval of $52.3 \mathrm{GHz}$. As the total gain bandwidth of Nd:YAG is estimated to be $120 \mathrm{GHz}$, this means that the cavity can generate two or three longitudinal modes at most. While, by applying relatively low pump power (about within 1.6 times of the threshold power) in the experiment, single longitudinal mode output could be obtained.

Since the LTO in our experiment is a-cut, the single frequency can be split into two frequencies with perpendicularly linear polarizations under its natural birefringence (the ordinary light polarized along the $\mathrm{x}$ axis, and the extraordinary light polarized along the $\mathrm{y}$ axis). The frequency difference of the two polarized light can be derived by the equation below:

$$
\Delta v=\frac{2 l_{L T O}}{\lambda}\left(n_{e}-n_{o}\right) \frac{c}{2 \mathrm{~L}} .
$$

where $n_{e}=2.1403, n_{o}=2.1363$ are the refractive indices of the ordinary and extraordinary light, respectively.

As the birefractive indexes of the LTO crystal is affected by temperature, the frequency difference has a correlationship with the cooling temperature of the microchip. The variation of the frequency difference with the cooling temperature is determined by the equation below [17]:

$$
\frac{d \Delta v}{d T}=\frac{2 l_{L T O}}{\lambda}\left[\frac{\vartheta n_{e}}{\vartheta T}-\frac{\vartheta n_{o}}{\vartheta T}+\alpha\left(n_{e}-n_{o}\right)\right] \frac{c}{2 L}
$$

where $\alpha$ is the expansion coefficient of LTO crystal in $\mathrm{z}$ direction. The value of parameters used in this paper are $\frac{\vartheta n_{o}}{\vartheta T}=25 \times 10^{-6} / \mathrm{K}, \frac{\vartheta n_{e}}{\vartheta T}=2.4 \times 10^{-6} / \mathrm{K}, \alpha=16 \times 10^{-6} / \mathrm{K}$ [17].

And as an electro-optic crystal, the birefractive indexes can also be tuned by electric filed. In the experiment, a transverse electric field is applied to the LTO crystal along the $\mathrm{x}$ axis. Due to the 
electro-optical effect, the refractive indexes of the LTO crystal along $\mathrm{x}$ and $\mathrm{y}$ axes are changed, and the changed value is proportional to the electric field strength and the electro-optic coefficient of LTO crystal.

$$
\Delta n_{o}=-\frac{\gamma_{13} n_{0}^{3} V}{2 d}, \Delta n_{e}=-\frac{\gamma_{33} n_{e}^{3} V}{2 d}
$$

Here $\gamma_{13}=8 \mathrm{pm} / V, \gamma_{33}=33 \mathrm{pm} / V, d=5 \mathrm{~mm}$, and $V$ is the applied voltage. The relationship between the voltage, the frequency difference of the ordinary light, and the extraordinary light is:

$$
\frac{d \Delta v}{d V}=\frac{l_{L T O}}{\lambda}\left[\frac{\gamma_{13} n_{0}^{3}-\gamma_{33} n_{e}^{3}}{d}\right] \frac{c}{2 L}
$$

Equations (3) and (5) give the theoretical relationships of $\Delta v$ with cooling temperature and applied voltage. For prediction of experiments results the slope tuning of the frequency difference versus temperature and voltage are calculated:

$$
\frac{d \Delta v}{d T}=2.36 \mathrm{GHz} / \mathrm{K}, \frac{d \Delta v}{d V}=2.56 \mathrm{MHz} / \mathrm{V}
$$

\section{Experimental Results and Discussion}

In our experiment, the lasing threshold of the microchip laser was found at around $900 \mathrm{~mW}$, at room temperature. At first, it was a single frequency output with x polarization. And when the pump power was increased, the y polarization $\mathrm{TEM}_{00}$ mode was generated, due to a relatively low spectral gain for the y polarization mode. If the pump power increased to exceed $1.5 \mathrm{~W}$, the neighboring longitudinal mode was generated. In the experiment, we obtained a dual-frequency laser output of about $34 \mathrm{~mW}$ at a pump power of $1.5 \mathrm{~W}$.

\subsection{Thermally Tunable Frequency Difference}

To roughly measure the frequency differences in a large range (about tens of $\mathrm{GHz}$ ) by tuning cooling temperature, a LSA IR-L optical spectrum analyzer with $0.02 \mathrm{~nm}$ resolution was used. The cooling temperature was continuously increased from $18{ }^{\circ} \mathrm{C}$ to $34{ }^{\circ} \mathrm{C}$ with a step interval of $0.5^{\circ} \mathrm{C}$. The measurement results are shown in Figure 2.

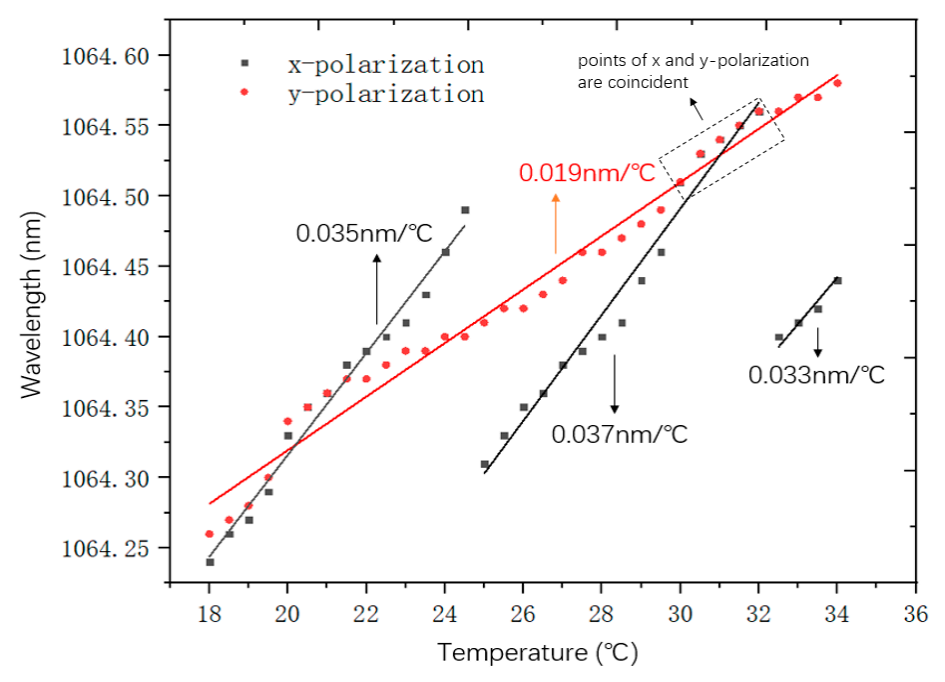

Figure 2. Variations of the wavelengths of the dual-frequency components with temperature increasing from $18{ }^{\circ} \mathrm{C}$ to $34^{\circ} \mathrm{C}$. The points of $\mathrm{x}$ - and y-polarization are approximately overlapped between $30^{\circ} \mathrm{C}$ and $32^{\circ} \mathrm{C}$. 
The $\mathrm{x}$ and $\mathrm{y}$ polarization correspond to the black and the red curves respectively. By fitting the data points, it can be seen that the laser wavelengths of the two polarizations vary linearly with the temperature increasing. It can be seen that, for y polarized wave, the wavelength curve is monotonic increasing from $1064.26 \mathrm{~nm}$ to $1064.58 \mathrm{~nm}$. While, for the x polarized wave curve, there are two saltus steps at the temperature around $25^{\circ} \mathrm{C}$ and $32{ }^{\circ} \mathrm{C}$. This is because of the longitudinal mode is hopping between neighbor longitudinal modes. It's affected by the relative positions of the gain curve and the cavity longitudinal modes. When the longitudinal mode's optical frequency shifts away from the center of the gain curve, its gain coefficient will drop. Frequency of the neighboring longitudinal mode will move closer to the center of the gain curve and obtain a larger gain coefficient. Due to mode competition, when the net gain of the latter is stronger than that of the former, the former will be extinguished. The mode hopping occurred at $24.5^{\circ} \mathrm{C}$, the wavelength changed from $1064.49 \mathrm{~nm}$ to $1064.31 \mathrm{~nm}$, coming with an approximate $54 \mathrm{GHz}$ frequency difference change, and the second one occurred at $31.5^{\circ} \mathrm{C}$, the wavelength changed from $1064.56 \mathrm{~nm}$ to $1064.39 \mathrm{~nm}$, with a $51 \mathrm{GHz}$ frequency difference change, approximately. They were both very close to the longitudinal mode interval of 52.3 GHz. While, it's interesting that there was no mode hoping for the y polarized wave in such a large frequency variation range, further investigation on this problem is needed in later works.

When the cooling temperature was between $18^{\circ} \mathrm{C}$ and $24.5^{\circ} \mathrm{C}$, the wavelengths of both the ordinary and the extraordinary beam continuously changed with temperature, and no mode hopping occurred. In this temperature range, we used an F-P scanning interferometer to measure the frequency differences more precisely. the F-P scanning interferometer continuously changes the transmission frequency by scanning voltage, ensuring that all frequencies could be transmitted and detected by its photodetector at the back end. Using the F-P scanning interferometer, the number of frequencies and frequency interval of the laser output can be measured. The measurement results are shown in Figure 3a. The results are fitted to obtain a good linear relationship between the frequency difference and the cooling temperature, with an average tuning slope of $4.4 \mathrm{GHz} /{ }^{\circ} \mathrm{C}$. It could be seen that there was a reverse between $20.5^{\circ} \mathrm{C}$ and $21^{\circ} \mathrm{C}$ during the temperature's variation. At $18^{\circ} \mathrm{C}$ to $20.5^{\circ} \mathrm{C}$, it was seen $v_{x}<v_{y}$, and at $21^{\circ} \mathrm{C}$ to $24.5^{\circ} \mathrm{C}$, it changed to $v_{x}>v_{y}$. The tuning slopes in the two temperature ranges were $-4.45 \mathrm{GHz} /{ }^{\circ} \mathrm{C}$ and $4.62 \mathrm{GHz} /{ }^{\circ} \mathrm{C}$, respectively. The average inaccuracy of the frequency difference for each point was about $\pm 50 \mathrm{MHz}$, due to the $\pm 0.01{ }^{\circ} \mathrm{C}$ accuracy of the temperature controller we used. The maximum frequency difference reached $27 \mathrm{GHz}$ at $24.5^{\circ} \mathrm{C}$.
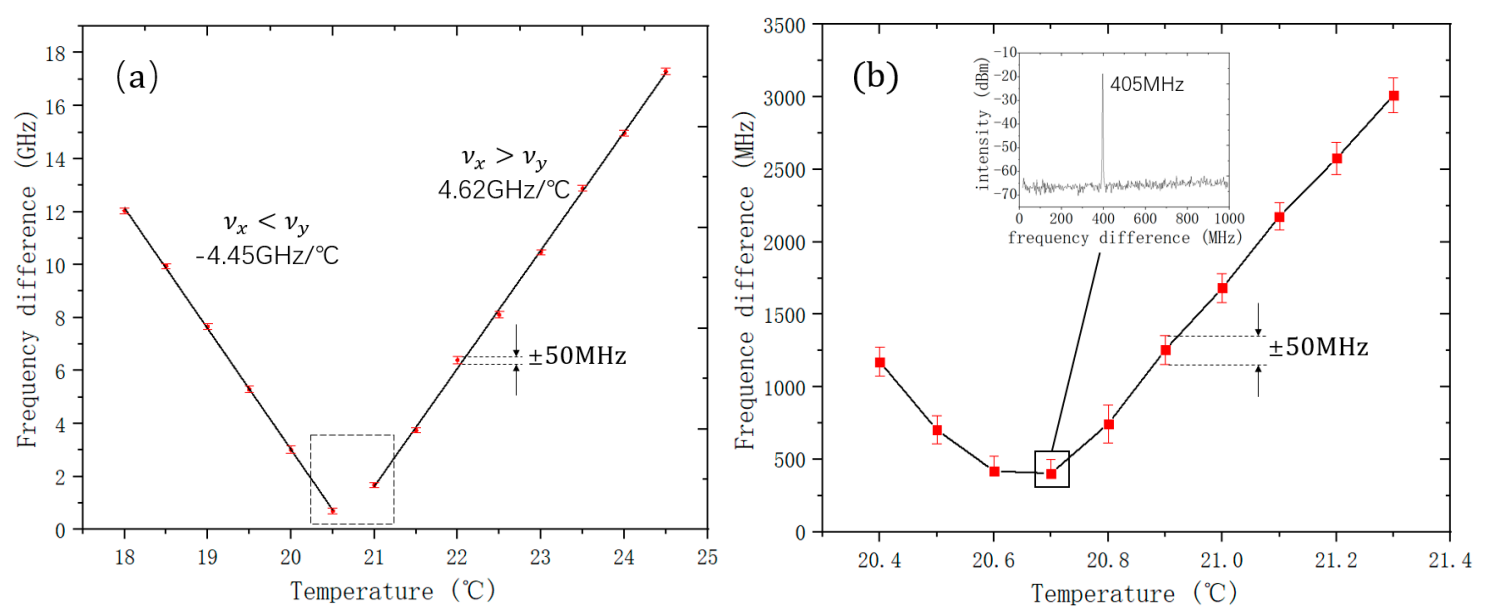

Figure 3. The relationship between the frequency difference and temperature of $18{ }^{\circ} \mathrm{C}-24.5^{\circ} \mathrm{C}(\mathrm{a})$ and $20.4^{\circ} \mathrm{C}-21.3^{\circ} \mathrm{C}(\mathbf{b})$. Data was measured by an F-P scanning interferometer. The minimum value of the frequency difference (in the insert of $(\mathbf{b})$ ) at $20.7^{\circ} \mathrm{C}$ was measured by a fiber input photon detector.

In the experiment, we observed that as the frequency of the two orthogonally polarized beams got quite close, one of them was extinguished, resulting in a single polarization output. This is a lock-in phenomenon of the two frequencies [18]. It is a ubiquitous phenomenon the orthogonally 
polarized dual-frequency lasers. Previous research on this phenomenon was focused on He-Ne lasers. The He-Ne dual-frequency laser based on the birefringent effect has a frequency difference lock-in of about $40 \mathrm{MHz}$ [19]. As far as we know, there is no report on the frequency of the lock-in phenomenon in solid-state dual-frequency lasers, while a quite low beat frequency can be achieved [20]. Considering the c-cut condition of the LTO crystal, the light oscillates parallel to the optical axis in the resonant cavity, and the frequency does not split due to the same refractive index for the a and $\mathrm{b}$ axes of LTO. If voltage or pressure is applied to change the direction and refractive index of the crystal's optical axis, the frequency difference will increase from a small value without a significant frequency difference lock-in effect [15]. In our experiment, the electro-optic crystal was a-cut, and the optical axis was perpendicular to the direction of light propagation. Due to natural birefringence, the frequency difference between two orthogonally polarized beams split by the same longitudinal mode is large at room temperature $\left(24^{\circ} \mathrm{C}\right)$, with a magnitude of more than $10 \mathrm{GHz}$. The minimum value of the frequency difference was measured during the continuous changing of the temperature. It can be seen from the measurement results in Figure 3a that the frequency difference continuously decreased as the temperature decreased, and the two frequencies should thus coincide between $20.5^{\circ} \mathrm{C}$ and $21^{\circ} \mathrm{C}$. Considering that the measurement accuracy is limited with an optical spectrum analyzer, the minimum value of the frequency difference cannot be accurately detected. Then, we switch to a RF spectrum analyzer with an InGaAs photon detector, its wavelength response range was 950-1630 nm and the bandwidth was $3.5 \mathrm{GHz}$. At $21.3^{\circ} \mathrm{C}$, a frequency difference of $3.0 \mathrm{GHz}$ was detected, and the temperature is continuously reduced. The curve of the frequency difference showed a process of folding back in the $20.6-20.8^{\circ} \mathrm{C}$ range, as shown in Figure $3 \mathrm{~b}$. This indicates that the frequency difference had passed through the minimum value of about $405 \mathrm{MHz}$. The results show that when the frequency difference dropped to $405 \mathrm{MHz}$, within a small range of temperature regulation $\left(0.1^{\circ} \mathrm{C}\right)$, the beat frequency signal disappeared. When the frequency difference was close to $405 \mathrm{MHz}$, by keeping the temperature constant and applying a voltage to change the frequency difference continuously, it was found that the beat frequency signal also disappeared below $405 \mathrm{MHz}$.

\subsection{Electrically Tunable Frequency Difference}

In order to achieve more precise tuning of the beat frequency, a high direct-voltage was applied to the electrode on the LTO crystal. The transverse electric field changed the refractive indexes of $b$ and c axes of LTO, and realized the tuning of the frequency difference. The relationship between the voltage and the frequency difference depends on Equation (5) in Section 3. With no voltage applied, a frequency difference of $0.7 \mathrm{GHz}$ was obtained at $20.8^{\circ} \mathrm{C}$, as shown in Figure $3 \mathrm{~b}$. When the voltage was applied, we could obtain the beat frequency versus the voltage, as shown in Figure 4 . The results were detected by the photodiode and measured by a RF spectrum analyzer. Due to the influence of controlling temperature accuracy, the frequency difference had the same fluctuation value as that in Figure 3. Here, we selected the median value of the float as the data point.

The measurement results showed that the curve of frequency difference vs. voltage changes linearly with a tuning slope of $1.23 \mathrm{MHz} / \mathrm{V}$. Considering that the accuracy of the applied voltage was $1.0 \mathrm{~V}$, it was more sensitive compared with controlling the temperature. On the other hand, the applied voltage not only tunes the frequency difference more accurately and quickly than the temperature, but also facilitates the stabilization of the frequency difference with a phase-locked loop [21]. Compared with the electro-optical coefficient $\gamma_{13}=8.6 \mathrm{pm} / \mathrm{V}, \gamma_{33}=30.8 \mathrm{pm} / \mathrm{V}$ of $\mathrm{LiNbO}_{3}$, the difference between the electro-optic coefficients $\gamma_{13}=8 \mathrm{pm} / \mathrm{V}$ and $\gamma_{33}=33 \mathrm{pm} / \mathrm{V}$ of $\mathrm{LiTaO}_{3}$ is larger. It can be seen from Equation (5) that this is more favorable for obtaining a wide range of tuning of the frequency difference. Theoretically, a similar maximum frequency difference to the temperature tuning can be achieved by increasing the applied voltage. However, due to the low sensitivity, if we want to achieve a frequency difference of up to about $20 \mathrm{GHz}$, an extremely high voltage of $16 \mathrm{kV}$ is required. Compared with the thermal tuning method, its cost is high and the processing difficulty of insulating the laser cavity is increased. So, large-scale thermal tuning of the frequency difference is needed. 


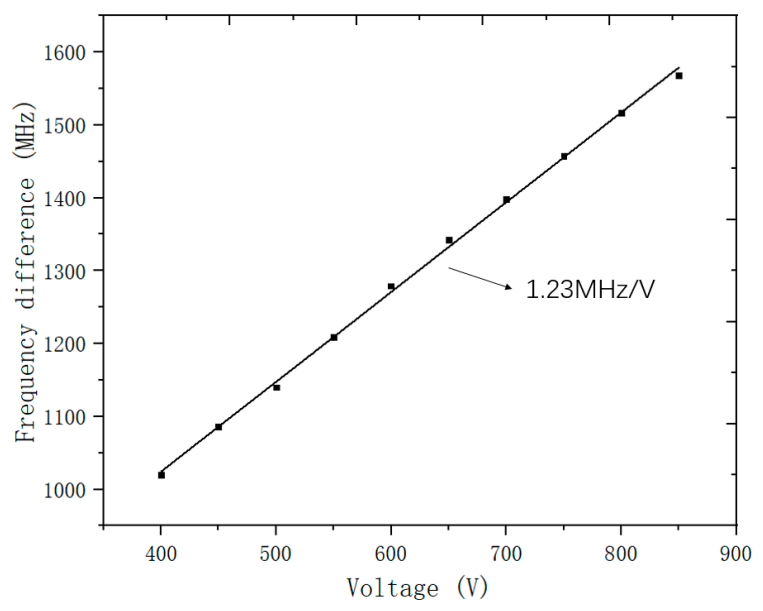

Figure 4. The relationship between the frequency difference and voltage when the temperature is constant.

\section{Conclusions}

A beat frequency tunable dual-frequency microchip laser is realized based on the thermo-optical and electro-optical effects of a LTO crystal. Through theoretical analysis, it is found that the temperature and voltage can be adjusted to tune the frequency difference at different scales. By combining the thermal and electrical tuning methods in an experiment, we demonstrated it can ensure broad-range and high-accuracy tuning of the frequency difference simultaneously. The frequency difference can be tuned by the temperature and voltage with a sensitivity of $4.5 \mathrm{GHz} /{ }^{\circ} \mathrm{C}$ and $1.23 \mathrm{MHz} / \mathrm{V}$, respectively. The maximum frequency difference achieved is about $27 \mathrm{GHz}$, and the minimum tunable frequency difference obtained is about $405 \mathrm{MHz}$ due to the lock-in effect of two frequencies.

Author Contributions: Conceptualization, C.Z., Z.Z. and K.G.; methodology, Z.Z. and K.G.; software, K.G. and Y.X.; validation, Z.Z., Y.X. and K.G.; formal analysis, K.G.; investigation, Z.Z., H.Z. and K.G.; resources, Z.Z., H.Z. and C.Z.; data curation, K.G.; writing—original draft preparation, K.G.; writing-review and editing, Z.Z. and K.G.; visualization, K.G.; supervision, Z.Z., H.Z. and C.Z.; project administration, Z.Z.; funding acquisition, Z.Z.

Funding: This research is funded by the National Natural Science Foundation of China, grant number 61805013.

Conflicts of Interest: The authors declare no conflict of interest.

\section{References}

1. Pillet, G.; Morvan, L.; Ménager, L.; Garcia, A.; Babiel, S.; Stöhr, A. Dual-Frequency Laser Phase Locked at 100 GHz. J. Lightw. Technol. 2014, 32, 3824-3830. [CrossRef]

2. Paquet, R.; Le Gratiet, L.; Sellahi, M.; Chomet, B.; Beaudoin, G.; Sagnes, I.; Garnache, A.; Blin, S.; Myara, M. Coherent continuous-wave dual-frequency high-Q external-cavity semiconductor laser for $\mathrm{GHz}-\mathrm{THz}$ applications. Opt. Lett. 2016, 41, 3751-3754. [CrossRef] [PubMed]

3. Brunel, M.; Lai, N.D.; Vallet, M.; Le Floch, A.; Bretenaker, F.; Morvan, L.; Dolfi, D.; Huignard, J.P.; Blanc, S.; Merlet, T. Generation of tunable high-purity microwave and terahertz signals by two-frequency solid state lasers. Proc. SPIE 2004, 5466. [CrossRef]

4. Hu, M.; Wei, M.; Zeng, R.; Li, Q.; Lu, Y.; Wei, Y.; Zhang, Y. Microchip dual-frequency laser with well-balanced intensity utilizing temperature control. Opt. Express 2016, 24, 23383-23389. [CrossRef] [PubMed]

5. Li, J.; Niu, Y.; Niu, H. Frequency difference stabilization in dual-frequency laser by stress-induced birefringence closed-loop control. Appl. Opt. 2016, 55, 4357-4361. [CrossRef] [PubMed]

6. Zhang, Z.; Gui, K.; Zhao, C.; Zhang, H.; Chen, S.; Zhou, G. Self-Q-switch regime based on a beat effect with a dual-frequency microchip laser. Phys. Rev. A 2018, 98, 033831. [CrossRef]

7. Yang, H.; Brunel, M.; Zhang, H.; Vallet, M.; Zhao, C.; Yang, S. RF Up-Conversion and Waveform Generation Using a Frequency-Shifting Amplifying Fiber Loop, Application to Doppler Velocimetry. IEEE Photonics J. 2017, 9, 1-9. [CrossRef] 
8. Chen, J.; Zhu, H.; Guo, D.; Hao, H.; Xia, W.; Wang, M. Self-mixing birefringent dual-frequency laser Doppler velocimeter. Opt. Express 2017, 25, 560-572. [CrossRef] [PubMed]

9. Onori, D.; Scotti, F.; Laghezza, F.; Scaffardi, M.; Bogoni, A. Coherent laser radar with dual-frequency Doppler estimation and interferometric range detection. In Proceedings of the Radar Conference (RadarConf), Philadelphia, PA, USA, 2-6 May 2016.

10. Zheng, X.; Zhao, C.; Zhang, H.; Zheng, Z.; Yang, H. Coherent dual-frequency lidar system design for distance and speed measurements. In Proceedings of the 2017 International Conference on Optical Instruments and Technology: Advanced Laser Technology and Applications, Beijing, China, 28-30 October 2017.

11. Mckay, A.; Dawes, J.M. Tunable Terahertz Signals Using a Helicoidally Polarized Ceramic Microchip Laser. IEEE Photonics Technol. Lett. 2009, 21, 480-482. [CrossRef]

12. Romanelli, M.; Wang, L.; Brunel, M.; Vallet, M. Measuring the universal synchronization properties of driven oscillators across a Hopf instability. Opt. Express 2014, 22, 7364-7373. [CrossRef] [PubMed]

13. Huang, C.N.; Li, Y.; Guo, H.; Zhu, J.; Zhang, S. Novel tunable dual-frequency laser with large frequency difference. Chin. J. Lasers 2002, 11, 229-231. [CrossRef]

14. Brunel, M.; Amon, A.; Vallet, M. Dual-polarization microchip lasers at 1.53 um. Opt. Lett. 2005, 30, 2418-2420. [CrossRef] [PubMed]

15. Qiao, Y.; Zheng, S.; Chi, H.; Jin, X.; Zhang, X. Electro-optically tunable microwave source based on composite-cavity microchip laser. Opt. Express 2012, 20, 29090-29095. [CrossRef] [PubMed]

16. Rolland, A.; Brunel, M.; Loas, G.; Frein, L.; Vallet, M.; Alouini, M. Beat note stabilization of a 10-60 GHz dual-polarization microlaser through optical down conversion. Opt. Express 2011, 19, 4399-4404. [CrossRef] [PubMed]

17. Rolland, A.; Frein, L.; Vallet, M.; Brunel, M.; Bondu, F.; Merlet, T. 40-GHz Photonic Synthesizer Using a Dual-Polarization Microlaser. IEEE Photonics Technol. Lett. 2010, 22, 1738-1740. [CrossRef]

18. Yang, S.; Zhang, S. The frequency split phenomenon in a He-Ne laser with a rotational quartz plate in its cavity. Opt. Commun. 1988, 68, 55-57. [CrossRef]

19. Jin, Y.Y.; Zhang, S.L.; Li, Y.; Guo, J.-H.; Li, J.-Q. Zeeman-birefringence He-Ne dual frequency laser. Chin. Phys. Lett. 2001, 18, 533-536. [CrossRef]

20. Gudelev, V.G.; Mashko, V.V.; Nikeenko, N.K.; Ryabtsev, G.I.; Stalmashonak, A.B.; Teplyashin, L.L. Diode-pumped cw tunable two-frequency YAG: $\mathrm{Nd}^{3+}$ laser with coupled resonators. Appl. Phys. B 2003, 76, 249-252. [CrossRef]

21. Rolland, A.; Loas, G.; Brunel, M.; Frein, L.; Vallet, M.; Alouini, M. Non-linear optoelectronic phase-locked loop for stabilization of opto-millimeter waves: Towards a narrow linewidth tunable THz source. Opt. Express 2011, 19, 17944-17950. [CrossRef] [PubMed]

(C) 2019 by the authors. Licensee MDPI, Basel, Switzerland. This article is an open access article distributed under the terms and conditions of the Creative Commons Attribution (CC BY) license (http://creativecommons.org/licenses/by/4.0/). 